\title{
Third Workshop on Teaching Computational Science (WTCS 2009)
}

\author{
Alfredo Tirado-Ramos ${ }^{1}$ and Angela Shiflet ${ }^{2}$ \\ ${ }^{1}$ Informatics Institute, University of Amsterdam \\ Amsterdam, The Netherlands \\ ${ }^{2}$ Wofford College \\ Spartanburg, South Carolina, U.S.A. \\ alfredo@science.uva.nl, shifletab@wofford.edu
}

\begin{abstract}
The Third Workshop on Teaching Computational Science, within the International Conference on Computational Science, provides a platform for discussing innovations in teaching computational sciences at all levels and contexts of higher education. This editorial provides an introduction to the work presented during the sessions.
\end{abstract}

Keywords: computational science, teaching, parallel computing, e-Learning, collaborative environments.

\section{Introduction}

Today's technology-driven societies require students who have been trained in technology-based environments, such as computational science. Until recently, computational science education was too costly and impractical for most academic institutions of higher learning. Now, however, such institutions can integrate methods from computer science, mathematical modeling, simulation, and scientific visualization, among others, to create virtual laboratories for in-silico experimentation and learning. The interaction of computational methods allows teachers and students to pose more intriguing questions in lower cost experimental settings. Thus, higher education is currently witnessing the rapid adoption of computational tools and methods by science teachers around the world. In the past few years, many teachers have shared experiences on the use of high performance, as well as not-so-high performance, computing facilities in order to promote the benefits and importance of computational science instruction in science classrooms. The Third International Workshop on Teaching Computational Science (WTCS2009), held in Baton Rouge, Louisiana, U.S.A., in conjunction with the International Conference on Computational Science (ICCS 2009) offers a technical program consisting of presentations dealing with the state of the art in the field, following the successful 2008 WTCS in Krakow, Poland [1]. The workshop includes presentations that describe innovations in the context of formal courses involving, for example, introductory programming, service courses and specialist undergraduate or postgraduate topics. 


\section{The Workshop}

During the workshop sessions, we had 12 oral and 2 poster presentations. Nguyen et al discussed interesting approach to statistical methods to infer knowledge during the learning process. Landau proposes that teaching within this research-like, problemsolving approach is a more motivating and efficient technique than teaching the various disciplines separately. Jacobs presents an interesting experience and provides access to web material, which could be useful to prepare other similar programs. Bloomfield describes the use of $\mathrm{R}$, a language typically used in computational science and engineering, as a useful tool in computational science teaching. Muresano describes a new innovative masters degree program with the aim of introducing students to core concepts such as large scale simulation and high performance computing. Shiflet describes successful strategies for managing epidemics in a microbiology course, where science students are usually unaware of potential advantages and complexities. Del Vado discusses an intelligent tutoring system for teaching data structures, an important subject in computational science training. Gavilanes describes an interesting tool for use in introductory classes, which allows students to focus on schemas and algorithm design rather than language syntax. Borne presents a computational science program centered on data representation and visualization covering a broad range of physical and biological sciences. Stevenson proposes extending classical argumentation structures as the basis for computational science education. Liu provides a summary of simulation and modeling process that illustrate how teaching tools can be used and how increasingly complex models can be introduced. Wallin presents the structure and goals of the first two years of a computational mathematics program, along with some observations about the elements that we have found that have been challenging in its implementation. Finally, as poster presentations, Gallardo presents an approach to adaptation to European Credit Transfer System, and Parker presents a new mathematics elective for an undergrad computational science program.

\section{Conclusions}

The presentations in ICCS 2009's Third International Workshop on Teaching Computational Science (WTCS2009) illustrate the variety and depth of computational science education around the world. Moreover, the growing success of the workshop attests to the increasing interest in this important interdisciplinary area.

Acknowledgments. We would like to acknowledge P.M.A. Sloot and D. van Albada for their continuous support and commitment to the success of this workshop.

\section{Reference}

1. Tirado-Ramos, A., Luo, Q.: Second Workshop on Teaching Computational Science WTCS 2008. In: Bubak, M., van Albada, G.D., Dongarra, J., Sloot, P.M.A. (eds.) ICCS 2008, Part II. LNCS, vol. 5102, pp. 657-658. Springer, Heidelberg (2008) 